\title{
Mapeamento Sistemático da Aplicabilidade de Materiais Autorregenerativos no Monitoramento Ativo e Online de Sistemas Mecânicos
}

\author{
Systematic Mapping of the Applicability of Self-healing Materials in the Active and Online \\ Monitoring of Mechanical Systems
}

Stanley Washington Ferreira de Rezende ${ }^{1}$, Bruno Pereira Barella², José dos Reis Vieira de Moura Júnior ${ }^{3}$

\section{RESUMO}

O método de monitoramento da integridade estrutural (SHM - do inglês Structural Health Monitoring) tem sido amplamente utilizado em processos de detecção, localização e quantificação de danos em sistemas mecânicos. Contudo, nos últimos anos houve um crescente busca por métodos que não apenas possibilitasse a identificação da presença de falha, mas que de certo modo interagisse ao sistema propondo medidas de contenção e reparo dos mesmos. Este trabalho apresenta uma revisão sistemática da literatura quanto ao desenvolvimento e a aplicabilidade de sistemas autorregenerativos no monitoramento ativo de falhas em componentes estruturais. Ademais, este estudo possui como foco também o atual estado da arte quanto a utilização de ligas de memória de forma como materiais de autocura. Para realização do mapeamento sistêmico, as bases de dados utilizadas foram: Portal de Periódicos da Coordenação de Aperfeiçoamento de Pessoal de Nível Superior (CAPES), Scopus, Web of Science e Engineering Village. Ressalta-se que a execução do processo de mapping study possibilitou a otimização do estudo realizado, bem como possibilitou a identificação dos principais GAP's referentes ao assunto abordado. Os resultados demonstraram que o processo de aplicação de materiais autorregenerativos em sistemas mecânicos se trata de um assunto recente com grandes possibilidades a serem ainda exploradas tais como a agregação de diferentes métodos de avaliação de danos, métodos estatísticos de inferência e sistemas remotamente controlados de forma a desenvolve-lo em meio online.

Palavras-chave: Monitoramento da Integridade Estrutural, Materiais Autorregenerativos, Sistemas de Auto-reparo Estrutural.

\section{ABSTRACT}

The Structural Health Monitoring (SHM) method has been widely used in the detection, localization, and quantification of mechanical system damage. However, in recent years there has been a growing search for methods that not only made it possible to identify the presence of failure, but which in a way interacted with the system by proposing measures to contain and repair them. This contribution presents a systematic review of the literature on the development and applicability of self-healing systems in the active monitoring of failures in structural components. In addition, this study also focuses on the current state of the art regarding the use of memory alloys as self-healing materials. To perform the systemic mapping, the databases used were: Portal of Periodicals of the Coordination of Improvement of Higher-Level Personnel (CAPES), Scopus, Web of Science and Engineering Village. It is noteworthy that the execution of the mapping study process made possible the optimization of the study, as well as the identification of the main GAPs related to the subject. Results demonstrated that the process of applying self-regenerative materials in mechanical systems is a recent subject with great possibilities to be explored, such as the aggregation of different damage assessment methods, statistical inference methods and remotely controlled systems of way to develop it online.

Keywords: Structural Health Monitoring, Self-healing materials, Self-repair systems.
Mestrando em Modelagem e Otimização - PPGMO.

IMTec - Unidade Acadêmica de Matemática e Tecnologia.

Universidade Federal de Goiás.

stanley-washington@hotmail.com

Mestrando em Modelagem e Otimização - PPGMO.

IMTec - Unidade Acadêmica de Matemática e Tecnologia.

Universidade Federal de Goiás. brunobarella@hotmail.com

Doutor em Engenharia Mecânica.

Professor do Programa de Mestrado em Modelagem e Otimização - PPGMO.

IMTec - Unidade Acadêmica de Matemática e Tecnologia.

Universidade Federal de Goiás. zereis@ufg.br 


\section{INTRODUÇAOO}

Em engenharia aplicada, diversos são os sistemas estruturais suscetíveis ao aparecimento de falhas, trincas ou danos, seja devido a sua própria funcionalidade ou até mesmo a localização de sua operação. A presença de danos em um componente estrutural, agregada à possíveis erros de projeto podem acarretar em grandes prejuízos financeiros e de recursos humanos (MOURA JR e STEFFEN JR, 2006).

Nesse sentido, a capacidade de monitorar o tempo de vida útil de certos mecanismos estruturais tem conquistado grande foco nos últimos anos, seja no âmbito industrial ou acadêmico, sendo conhecida por Structural Health Monitoring (SHM). Essa área de estudo visa, principalmente, analisar a integridade estrutural dos sistemas mecânicos abrangendo não somente o processo de identificação do dano, mas também ações de correção e controle (FREl et al., 2013).

Ao mesmo passo, materiais com propriedades de autocura e os sistemas de autoreparo estrutural ativo têm apresentado grande usabilidade no desenvolvimento e construção de novos mecanismos industriais (HURLEBAUS e GAUL, 2006). Isto se deve ao fato, desses tipos de materiais possuírem características intrínsecas que permitem ações de restauração do sistema como um todo, sendo esta, realizada após uma condição de dano ser identificada (PEAIRS, PARK e INMAN, 2002; GHOSH et al., 2007; LESTER et al., 2015).

Segundo Kim et al. (2009) os sistemas de auto-reparo estrutural bem como a integração de materiais autorregenerativos nesses sistemas, representam-se no mais alto nível de monitoramento da integridade estrutural. Logo, tendo em vista o posterior aperfeiçoamento da área, variados projetos estruturais que implementam o conceito de autocura têm sido desenvolvidos nos últimos anos (BAROUDI et al., 2014; BRAGA et al., 2014).

As conexões parafusadas representam um dos principais componentes estruturais existentes nos sistemas mecânicos (RITDUMRONGKUL et al., 2003). Essas, invariavelmente, promovem o aparecimento e crescimento de falhas e, muitas das vezes, a complexidade de sua geometria e/ou a aplicação de cargas pontuais sobre elas dificultam ou impossibilitam o seu processo de inspeção e manutenção.

Em particular, as juntas aparafusadas se caracterizam por serem suscetíveis ao auto-afrouxamento de seus componentes. Além disso, essas também são sujeitas ao aparecimento de trincas por tração ou cisalhamento. Tais condições podem alterar o 
modo como estas estruturas respondem a um dado estimulo mecânico (WANG e WU, 2011).

Nesse seguimento, objetivando-se reduzir esse módulo de falha, estudos foram desenvolvidos buscando implementar o conceito de junta auto-sensitiva e auto-reparadora (Park e Inman, 2001; Peairs, Park e Inman, 2002).

Em seu trabalho, Park, Muntges e Inman (2003) realizaram a modelagem da quantidade de energia necessária para atuar anéis de liga de memória de forma (Shape Memory Alloy - SMA) acopladas a juntas aparafusadas, no intuito de restaurar o torque perdido. Contudo, devido a fatores como geometria complexa, conexões de múltiplos membros, leis constitutivas complicadas e desempenho inesperado da tinta sensível à temperatura, tais autores não encontraram um valor exato da energia necessária para aquecer completamente o mecanismo.

Kim et al. (2009) investigaram um processo de autorregulagem da perda de torque de parafusos, originado mediante o afrouxamento de juntas parafusadas. Para tanto, anilhas compostas por SMA foram aquecidas e expandidas axialmente a fim de se pressionar as diferentes partes do sistema mecânico. Chang, Lee e Park (2011) reproduziram esse experimento em vigas parafusadas, com o emprego de porcas compostas por liga de memória de forma. Para isso, utilizaram o método de SHM por impedância eletromecânica e pastilhas PZT para excitar a estrutura e verificar a presença de danos.

No trabalho desenvolvido por Shvetashva et al. (2016) foi verificado a capacidade de reparo de trincas em chapas de zinco compósitas por materiais de características de autocura. Na fase de identificação e localização do dano, foi utilizado o método de SHM baseado em Ondas de Lamb, e posteriormente o material autorregenerativo foi aquecido localmente afim de restaurar a região danificada.

Ademais, outras diversas configurações de SHM utilizando compostos de SMA como material autorregenerativo em juntas aparafusadas, podem ser encontrados em Hesse, Ghorashi e Inman (2004), Ghorashi e Inman (2004), Peairs, Park e Inman (2004), Antonios, Inman e Smaili (2006), Afshari, Marquié e Inman (2009), Garcia e Sodano (2010), Faria, Lopes Junior e Inman (2011).

Ainda, o aperfeiçoamento das técnicas de SHM em módulos online e sua posterior junção à mecanismos de auto-reparo estrutural possibilitam o desenvolvimento de sistemas restritos de manutenção, reduzindo-se assim os custos de operação. 
À face do exposto, o presente trabalho tem por objetivo apresentar o atual panorama de pesquisas que vêm sendo desenvolvidas sobre a aplicabilidade dos materiais autorregenerativos e dos ideais de sistemas de auto-reparo no monitoramento ativo e online de sistemas mecânicos.

\section{MATERIAIS E METODOS}

As fases de elaboração deste artigo de revisão se fundamentaram na metodologia de mapeamento de pesquisas proposta por Bailey et al. (2007) e Viana, Formoso e Kalsaas (2012). Essa por si consiste do levantamento sistemático da literatura que se conjectura em uma estrutura de busca por periódicos, mineração e análise de dados (BAILEY et al., 2007).

A revisão sistemática da literatura, também conhecida como mapping study, permite identificar a consistência de estudos existentes sobre determinado assunto, porém não se limitando apenas a reprodução dos resultados (COOPER, 2016). Assim sendo, o mapeamento realizado neste trabalho se baseou na pesquisa em bases de dados que possuem uma vasta quantidade de publicações e ferramentas de buscas, as quais possibilita o uso de expressões-chave para a seleção dos artigos.

Utilizou-se ao total quatro bases de dados, a saber: Scopus, Web of Science, Engineering Village e Portal de Periódicos da Coordenação de Aperfeiçoamento de Pessoal de Nível Superior (CAPES). Embora existam outras bases de dados, a pesquisa limitou-se somente a essas, visto que elas já são capazes de contemplar um número significativo de artigos e periódicos.

Ademais, foi empregado quatro expressões lógicas para à busca de periódicos que abordassem o uso de materiais autorregenerativos no monitoramento ativo de juntas mecânicas, que são elas:

$\checkmark$ EL1 - "Self-healing AND Structural Health Monitoring AND Shape Memory Alloy";

$\checkmark$ EL2 - "Bolted Joints AND Self-healing";

$\checkmark$ EL3 - "Bolted Joints AND Self-healing AND Shape Memory Alloy";

$\checkmark$ EL4 - "Bolted Joints AND Self-healing AND Structural Health Monitoring".

Em geral, o processo de busca se restringiu somente à artigos de periódicos em língua inglesa cujas expressões lógicas estavam presentes no título, no resumo e/ou nas palavras-chave. Aplicados os filtros em cada base de dados, os artigos foram então 
classificados pelo título e ano de publicação, eliminando-se ao mesmo tempo as possíveis repetições entre as bases de dados.

Após a primeira fase de coleta, uma base de dados foi criada no gerente de bibliografias JabRef de modo a excluir as possíveis duplicatas entre as distintas expressões-chave utilizadas e, subsequentemente, tornar a disposição dos dados mais intuitiva.

Posteriormente, em uma terceira etapa de filtragem realizou-se a leitura prévia dos resumos. Essa por si, teve a finalidade de excluir os múltiplos trabalhos que não se relacionavam diretamente ao tema proposto. Ainda, os artigos que não possuíam acesso gratuito pelo IP do sistema de biblioteca da Universidade Federal de Goiás - Regional Catalão (UFG) foram igualmente desconsiderados.

Objetivando-se tornar o mapping study desenvolvido mais robusto, buscou-se em uma última etapa por artigos de referência na área e que por ventura não foram identificados ou selecionados no mapeamento sistemático, sendo esta etapa denominada por snowball sampling.

A etapa de snowball foi realizada de maneira qualitativa, por meio da análise de referências utilizadas nos artigos classificados como aderentes em fases anteriores. Os dados finais foram então organizados e os resultados resumidos em forma de tabelas e gráficos sendo a revisão da bibliografia desenvolvida a partir do número final de estudos obtido.

\section{RESULTADOS E DISCUSSÃO}

Durante as etapas de mapeamento e filtragem, os resultados parciais encontrados foram armazenados em forma de gráficos e tabelas com o objetivo de tornar a sua compreensão mais sucinta e prática. A Tabela 1 apresenta a quantidade de artigos de periódicos encontrados para cada fase em relação as bases de dados utilizadas.

A partir da Tabela 1, observa-se que a string "Self-healing AND Structural Health Monitoring AND Shape Memory Alloy" foi a que teve maior retorno de artigos no mapeamento realizado. Contudo, a mesma também se caracterizou por possuir a maior quantidade de repetições entre as múltiplas palavras-chave.

A Figura 1 apresenta a relação final entre as expressões-chave utilizadas após a fase de remoção de duplicatas e leitura dos resumos. 
Tabela 1. Resumo dos resultados obtidos entre cada base de dados e expressões-chave.

\begin{tabular}{|c|c|c|c|c|c|c|c|c|}
\hline \multirow[t]{3}{*}{ Base de Dados } & \multicolumn{2}{|r|}{ EL1 } & \multicolumn{2}{|r|}{ EL2 } & \multicolumn{2}{|r|}{ EL3 } & \multicolumn{2}{|r|}{ EL4 } \\
\hline & \multicolumn{8}{|c|}{ Número de Artigos } \\
\hline & Inicial & $\begin{array}{l}\text { Com títulos } \\
\text { aderentes }\end{array}$ & Inicial & $\begin{array}{l}\text { Com títulos } \\
\text { aderentes }\end{array}$ & Inicial & $\begin{array}{l}\text { Com títulos } \\
\text { aderentes }\end{array}$ & Inicial & $\begin{array}{c}\text { Com } \\
\text { títulos } \\
\text { aderentes }\end{array}$ \\
\hline CAPES & 108 & 28 & 45 & 8 & 16 & 7 & 21 & 6 \\
\hline Scopus & 9 & 9 & 8 & 8 & 6 & 6 & 3 & 3 \\
\hline Web of Science & 4 & 4 & 7 & 7 & 7 & 7 & 5 & 2 \\
\hline Engineering Village & 10 & 10 & 7 & 7 & 7 & 7 & 7 & 7 \\
\hline Total Parcial & 131 & 51 & 67 & 30 & 36 & 27 & 36 & 18 \\
\hline $\begin{array}{l}\text { Número de artigos sem } \\
\text { repetições entre } \\
\text { base de dados }\end{array}$ & \multicolumn{2}{|r|}{36} & \multicolumn{2}{|r|}{10} & \multicolumn{2}{|r|}{9} & \multicolumn{2}{|r|}{12} \\
\hline $\begin{array}{l}\text { Número de artigos sem } \\
\text { repetições entre } \\
\text { expressões- chave }\end{array}$ & \multicolumn{8}{|c|}{24} \\
\hline $\begin{array}{l}\text { Número de artigos } \\
\text { selecionados após leitura do } \\
\text { resumo }\end{array}$ & \multicolumn{8}{|c|}{21} \\
\hline Snowball Sampling & \multicolumn{8}{|c|}{25} \\
\hline
\end{tabular}

Fonte: O autor (2020). 
Bolted Joints AND Self-healing AND Structural Health Monitoring

Bolted Joints AND Self-healing AND Shape Memory Alloy

Bolted Joints AND Self-healing

Self-healing AND Structural Health Monitoring AND Shape Memory Alloy
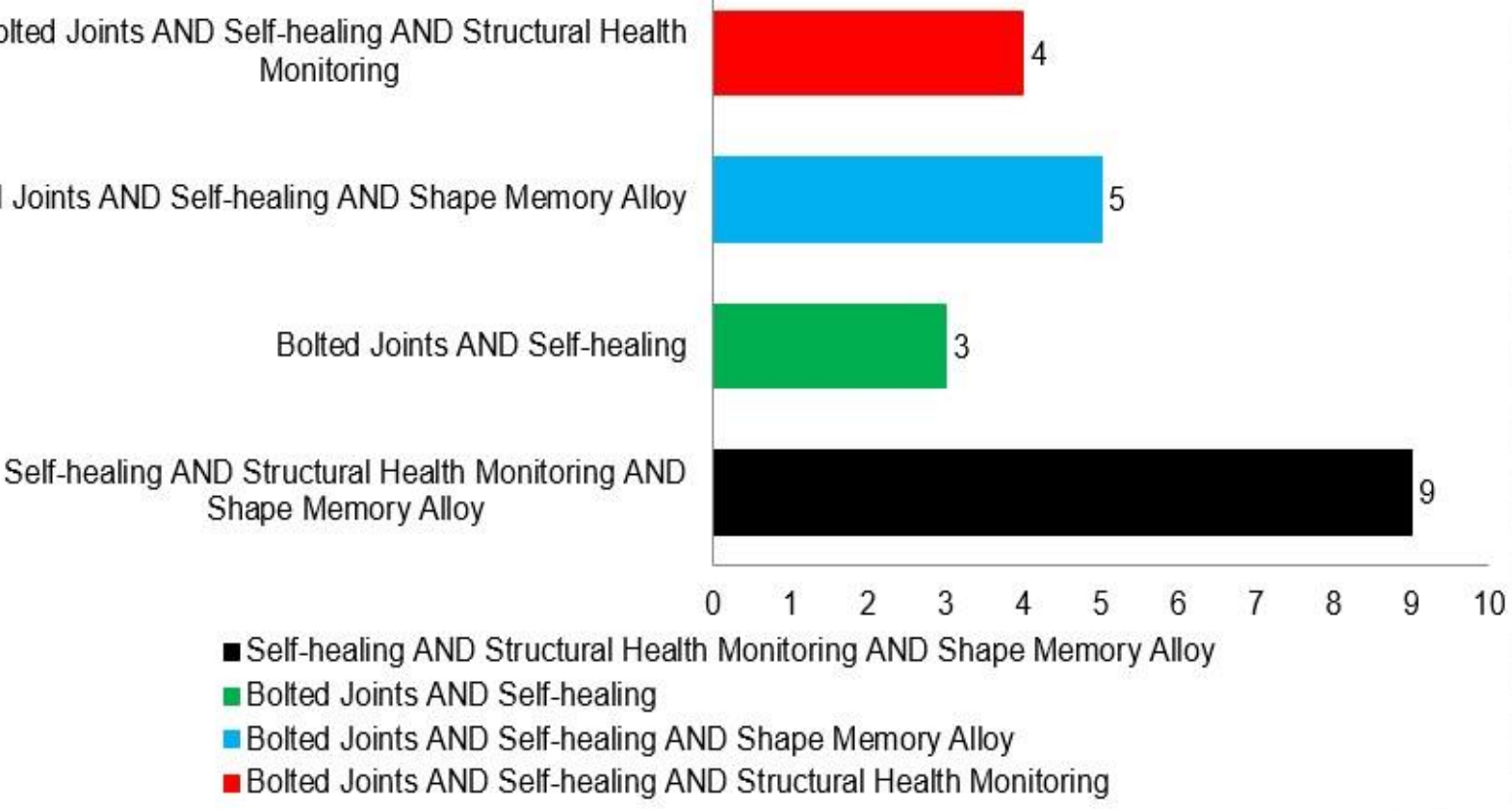

Figura 1. Relação entre a quantidade de artigos mapeados e as expressões-chave utilizadas.

Por intermédio da revisão sistêmica da literatura verificou-se que o tratamento realizado possibilitou uma filtragem significativa dos trabalhos, contribuindo para a otimização do estudo. A Tabela 2 exibe a relação da redução dos artigos analisados em cada etapa.

Tabela 2. Relação dos resultados parciais obtidos.

\begin{tabular}{c|c|c|c}
\cline { 2 - 4 } & Quantidade de Artigos & $\%$ Mantida & \% Redução \\
\hline Quantidade Inicial & 270 & $100 \%$ & $-0 \%$ \\
\hline Quantidade após 1\% Fase & 126 & $47 \%$ & $-53 \%$ \\
\hline Quantidade após 2\% Fase & 67 & $53 \%$ & $-47 \%$ \\
\hline Quantidade após 3\% Fase & 24 & $36 \%$ & $-64 \%$ \\
\hline Quantidade após 4\% Fase & 21 & $87,5 \%$ & $-12,5 \%$ \\
\hline Snowball Sampling & 25 & $119 \%$ & $+31 \%$ \\
\hline
\end{tabular}

Fonte: $O$ autor (2020).

Ao final do tratamento aplicado, um banco de dados com 25 referências aderentes ao tema foi elaborado. Essas, posteriormente, foram agrupadas de acordo com o seu período de publicação a fim de se compreender o processo de evolução de cada expressão-chave utilizada.

A Figura 2 apresenta a evolução das publicações dos artigos em periódicos, referentes a cada palavra-chave limitando-se a março de 2020. 


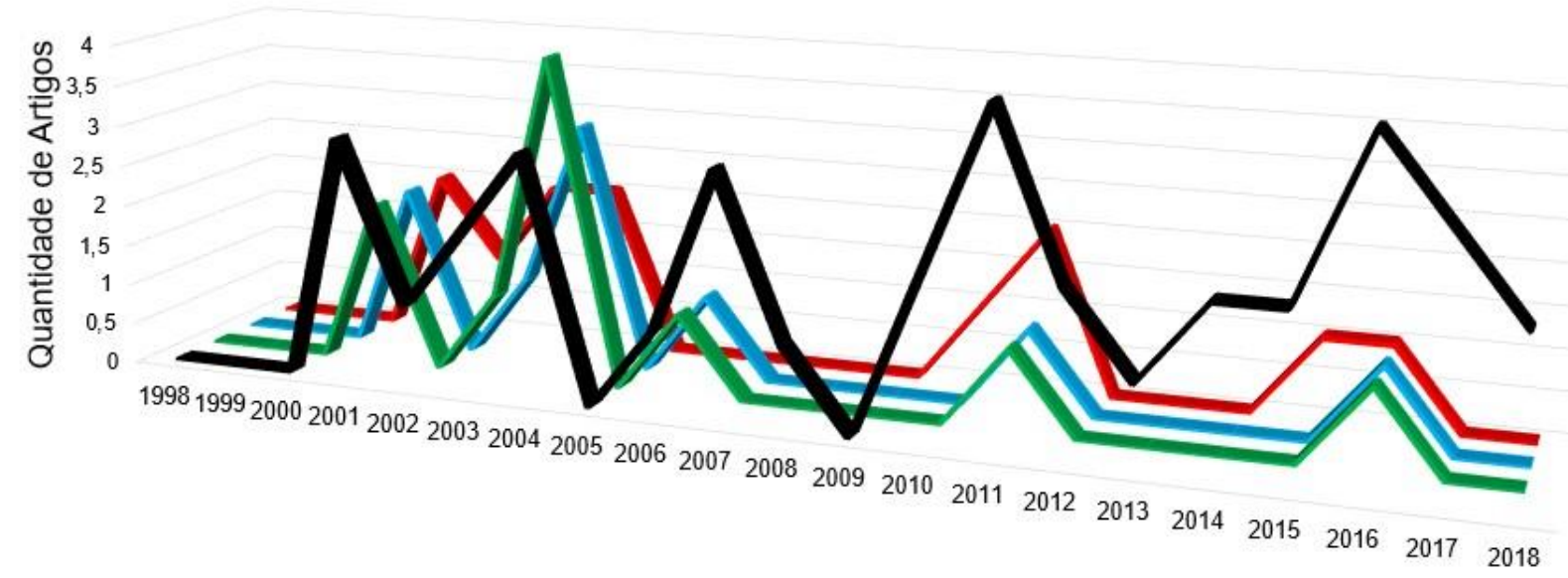

Período de Publicação

- Self-healing AND Structural Health Monitoring AND Shape Memory Alloy
Bolted Joints AND Self-healing
Bolted Joints AND Self-healing AND Shape Memory Alloy
- Bolted Joints AND Self-healing AND Structural Health Monitoring

Figura 2. Evolução das publicações dos artigos levantados em função das expressões chave utilizadas no mapeamento sistemático.

Nota-se pela Figura 2, que os artigos encontrados sobre o assunto abordado foram publicados nas últimas duas décadas, sendo o ano de 2001 o marco inicial para as publicações de periódicos. Através da leitura e análise dos trabalhos mapeados, percebese que se trata de um tema com grandes possibilidades e campos a serem explorados.

É importante ressaltar que, dentre os dados utilizados para contagem histórica, não foram contabilizados os trabalhos encontrados na fase de snowball sampling. Os principais periódicos empregados para publicações dos artigos podem ser visualizados na Tabela 3.

Tabela 3. Principais periódicos internacionais com publicações sobre o tema tratado.

\begin{tabular}{c|c}
\hline Periódico & № de artigos \\
\hline Acta Mechanica & 1 \\
\hline Decision Support Systems & 1 \\
\hline International Journal of Distributed Sensor Networks & 1 \\
\hline Journal of Operations Management & 1 \\
\hline Journal of Intelligent Material Systems and Structures & 4 \\
\hline Mechanical Systems and Signal Processing & 1 \\
\hline Proceedings of SPIE - The International Society for Optical Engineering & 2 \\
\hline Smart Materials and Structures & 3 \\
\hline
\end{tabular}




\section{Fonte: O autor (2020).}

O periódico Journal of Intelligent Material Systems and Structures foi o que apresentou a maior quantidade de publicações aderentes ao tema, possuindo ao todo 4 trabalhos publicados entre os anos de 2004 a 2011. A Figura 3 apresenta os principais países que possuem centros de pesquisas sobre o tema: aplicação de materiais autorregenerativos no monitoramento ativo de sistemas mecânicos.

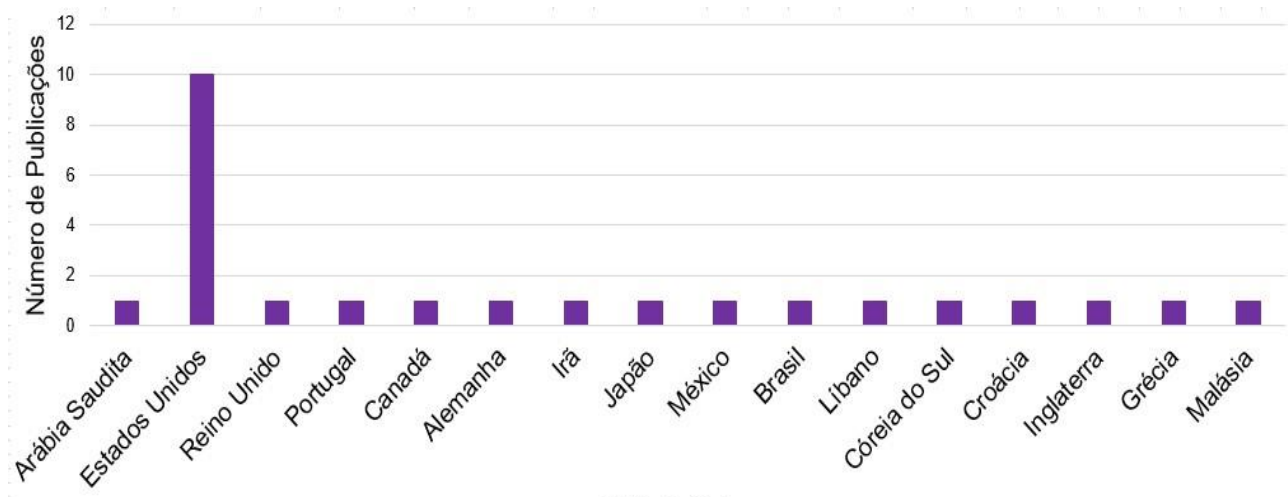

País de Origem

Figura 3. Principais países, entre os artigos selecionados, com publicações sobre o tema abordado.

Com base na Figura 3, observa-se que o Estados Unidos é o maior centro de pesquisa referente ao tema proposto, possuindo expressamente a maior quantidade de artigos publicados dentre os que foram selecionados. Ainda, nota-se que o Brasil possui apenas um único trabalho publicado sobre o tema, demonstrando uma carência de pesquisas referente a este assunto.

Na Tabela 4 é apresentado os principais pesquisadores relacionados às aplicações de materiais autorregenerativos em sistemas mecânicos. Destaca-se que, nesta análise foram considerados apenas o primeiro autor de cada artigo de periódico.

Tabela 4. Principais pesquisadores com artigo dentre o tópico aplicação de materiais autorregenerativos em sistemas mecânicos.

\begin{tabular}{c|c|c}
\hline Pesquisador & № de artigos & Período \\
\hline Afshari, M. & 1 & 2010 \\
\hline Antonios, C. & 1 & 2006 \\
\hline Baroudi, U. & 1 & 2014 \\
\hline Braga, D. F.O. & 1 & 2014 \\
\hline Chang, H. & 1 & 2011 \\
\hline
\end{tabular}




\begin{tabular}{c|c|c}
\hline Faria, C. T. & 1 & 2011 \\
\hline Frei, R. & 1 & 2013 \\
\hline Garcia, M. E. & 1 & 2010 \\
\hline Ghorashi, M. & 1 & 2004 \\
\hline Ghosh, D. & 1 & 2007 \\
\hline Hesse, T. & 1 & 2004 \\
\hline Hurlebaus, S. & 1 & 2006 \\
\hline Lester, B. & 1 & 2015 \\
\hline Park, G. & 2 & 2001 e 2003 \\
\hline Peairs, D. M. & 3 & 2002 a 2004 \\
\hline Ritdumrongkul, S. & 1 & 2004 \\
\hline Shvetashva, S. & 1 & 2016 \\
\hline Wang, Q. & 1 & 2012 \\
\hline
\end{tabular}

Fonte: O autor (2020).

A partir do exposto, percebe-se que os pesquisadores Daniel Peairs e Gyuhae Park representam cerca de $20 \%$ de todos os trabalhos referentes ao tema, sendo estes os introdutores do assunto em ambientes de pesquisa.

Após todas as etapas de filtragem serem realizadas e a partir da leitura dos artigos selecionados ser executada, pode-se perceber 5 vertentes principais adotadas pelos pesquisadores do assunto. A relação entre as vertentes identificadas e a quantidade de artigos de periódicos publicados é apresentada na Figura 4.

Utilização de polímeros SMA e fibra óptica para restauração de estrutura polimérica

\section{Utilização de SMA, pastilhas PZT e \\ do método de impedância \\ eletromecânica para restauração de \\ torque em juntas aparafusadas}

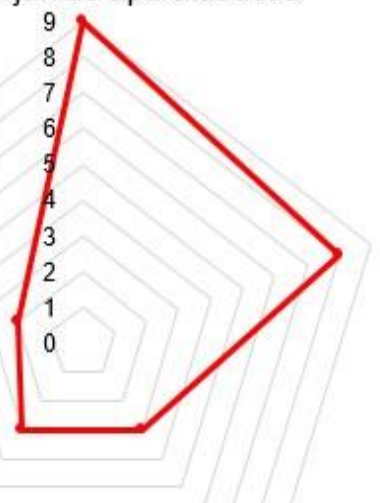

Estudo das propriedades de Liga de Memória de Forma

Identificação de danos por método de impedância eletromecânica
Estudos sobre processos de autocura

Figura 4. Principais vertentes encontradas nos artigos selecionados. 
Percebe-se que a pesquisa sobre a aplicabilidade dos materiais autorregenerativos e ideais de sistemas de auto-reparo estrutural no monitoramento ativo de sistemas mecânicos possibilita uma ampla gama de temas a serem explorados. Esses, se originam desde estudos introdutórios das propriedades básicas dos materiais autorregenerativos até a sua aplicação em práticas de monitoramento de estruturas reais (HURLEBAUS e GAUL, 2006; SHVETASHVA et al., 2016).

Foram encontrados ao total 9 trabalhos que se utilizam de ligas de memória de forma (SMA) e de pastilhas PZT para desenvolverem sistemas de auto-reparo de juntas aparafusadas, 8 trabalhos verificando as propriedades das ligas de memória de forma como material autorregenerativo, 3 trabalhos verificando tanto os processos de identificação de danos quanto processos de autocura e apenas 1 aplicando SMA em fibras poliméricas. Representando assim em forma de porcentagem cerca de $36 \%, 32 \%$, $12 \%, 12 \%$ e $8 \%$, respectivamente, de todos os trabalhos analisados.

Ressalta-se que poucos métodos foram encontrados quanto a aplicação dos materiais autorregenerativos em estruturas mecânicas reais, bem como o seu estudo em outros tipos de sistemas. Indicando assim que esta técnica pode ser investigada de maneira mais aprofundada a fim de se descobrir melhor suas vantagens e desvantagens com relação aos outros métodos utilizados com maior frequência pela literatura.

\section{CONSIDERACÓES FINAIS}

A partir do levantamento bibliográfico realizado neste trabalho, foi possível verificar a expansão dos estudos e aplicações de materiais autorregenerativos e desenvolvimento dos sistemas de auto-reparo estrutural no monitoramento ativo de sistemas mecânicos.

Ainda, pode-se avaliar que embora existam alguns estudos quanto aplicação prática desses tipos de materiais em estruturas do cotidiano industrial. Poucos métodos de avaliação de integridade foram utilizados para validação e identificação da presença de dano, bem como a integração da técnica já desenvolvida a outros sistemas de monitoramento, ativação e reparo estrutural.

Ademais, nota-se que apenas um tipo de material, sendo este a liga de memória de forma (SMA), foi efetivamente estudado quanto a sua aplicação no processo de selfhealing. Nessa linha de raciocínio, é possível destacar que embora já introduzido o tema em ambientes de pesquisa o mesmo possui alguns GAP's que necessitam de melhor exploração e aperfeiçoamento, como a sua integração a sistemas controlados remotamente de forma a desenvolver um processo de auto-reparo inteligente e online. 
Por fim, cabe destacar que foi empregado um método de mapeamento sistemático da literatura na elaboração dessa revisão, permitindo assim identificar as principais lacunas para o desenvolvimento de novas pesquisas. Principalmente, no direcionamento para as principais publicações ligadas ao estudo.

\section{AGRADECIMENTOS}

B. P. B. e S. W. F. R. agradecem a CAPES por suas bolsas de mestrado.

\section{REFERÉNCIAS}

AFSHARI, M.; MARQUIÉ, T. e INMAN, D. J. Automated structural health monitoring of bolted joints in railroad switches. In: ASME 2009 Rail Transportation Division Fall Technical Conference. American Society of Mechanical Engineers, p. 1-7. 2009.

ANTONIOS, C.; INMAN, D. J. e SMAILI, A. Experimental and theoretical behavior of selfhealing bolted joints. Journal of Intelligent Material Systems and Structures, v. 17, n. 6, p. 499-509. 2006.

BAILEY, J. et al. Evidence relating to object-oriented software design: A survey. In: IEEE, p. 482-484. 2007.

BAROUDI, U. et al. Smart bolts monitoring using wireless sensor network: implementation and performance evaluation. International Journal of Distributed Sensor Networks, v. 10, n. 6 , p. 546517.2014.

BRAGA, D. F. et al. Advanced design for lightweight structures: Review and prospects. Progress in Aerospace Sciences, v. 69, p. 29-39. 2014.

CHANG, H.; LEE, C. e PARK, S. Self-monitoring and self-healing bolted joints using shape memory alloy. In: 28th international symposium on automation and robotics in construction. p. 824-825. 2011.

COOPER, I. D. What is a "mapping study"?. Journal of the Medical Library Association: JMLA, Medical Library Association, v. 104, n. 1, p. 76. 2016.

FARIA, C. T.; LOPES JUNIOR, V. e INMAN, D. J. Modeling and experimental aspects of self-healing bolted joint through shape memory alloy actuators. Journal of Intelligent Material Systems and Structures, v. 22, n. 14, p. 1581-1594. 2011.

FREI, R. et al. Self-healing and self-repairing technologies. The International Journal of Advanced Manufacturing Technology, v. 69, n. 5-8, p. 1033-1061. 2013.

GARCIA, M. E. e SODANO, H. A. Biologically inspired autonomous structural materials with controlled toughening and healing. In: Active and Passive Smart Structures and Integrated Systems 2010. International Society for Optics and Photonics, p. 76431Z. 2010. 
GHORASHI, M. e INMAN, D. J. Shape memory alloy in tension and compression and its application as clamping force actuator in a bolted joint: part 2-modeling. Journal of intelligent material systems and structures, v. 15, n. 8, p. 589-600. 2004.

GHOSH, D. et al. Self-healing systems - survey and synthesis. Decision support systems, v. 42, n. 4, p. 2164-2185. 2007.

HESSE, T.; GHORASHI, M. e INMAN, D. J. Shape memory alloy in tension and compression and its application as clamping-force actuator in a bolted joint: part 1 experimentation. Journal of intelligent material systems and structures, v. 15, n. 8, p. 577-587. 2004.

HURLEBAUS, S. e GAUL, L. Smart structure dynamics. Mechanical Systems and Signal Processing, v. 20, n. 2, p. 255-281. 2006.

KIM, J. K. et al. A structural health monitoring system for self-repairing. In: Health Monitoring of Structural and Biological Systems 2009. International Society for Optics and Photonics, p. 729512. 2009.

LESTER, B. T. et al. Review and Perspectives: Shape memory alloy composite systems. Acta Mechanica, v. 226, n. 12, p. 3907-3960. 2015.

MOURA JR, J. R. V. e STEFFEN JR, V. Impedance-based health monitoring for aeronautic structures using statistical meta-modeling. Journal of intelligent material systems and structures, v. 17, n. 11, p. 1023-1036. 2006.

PARK, G. e INMAN, D. J. Smart bolts: an example of self-healing structures. Smart Materials Bulletin, v. 2001, n. 7, p. 5-8. 2001.

PARK, G.; MUNTGES, D. E. e INMAN, D. J. Self-repairing joints employing shapememory alloy actuators. JOM, v. 55, n. 12, p. 33-37. 2003.

PEAIRS, D. M.; PARK, G. e INMAN, D. J. Self-healing bolted joint analysis. Hand, v. 10, n. 1, p. 100. 2002.

PEAIRS, D. M.; PARK, G. e INMAN, D. J. Practical issues in self-repairing bolted joints. In: Smart Structures and Materials 2003: Smart Structures and Integrated Systems. International Society for Optics and Photonics, p. 81-93. 2003.

PEAIRS, D. M.; PARK, G. e INMAN, D. J. Practical issues of activating self-repairing bolted joints. Smart Materials and Structures, v. 13, n. 6, p. 1414. 2004.

RITDUMRONGKUL, S. et al. Quantitative health monitoring of bolted joints using a piezoceramic actuator-sensor. Smart materials and structures, v. 13, n. 1, p. 20. 2003.

SHVETASHVA, S. U. R. I. et al. Initial integration of ultrasonic structural health monitoring of self-healing materials. 2016.

VIANA, D. D.; FORMOSO, C. T.; KALSAAS, B. T. Waste in construction: a systematic literature review on empirical studies. In: ID Tommelein \& CL Pasquire, 20th Annual 
Conference of the International Group for Lean Construction. San Diego, USA. p. 18-20. 2012.

WANG, Q. e WU, N. A review on structural enhancement and repair using piezoelectric materials and shape memory alloys. Smart Materials and Structures, v. 21, n. 1, p. 013001. 2011. 